\title{
Managing suspected temporal arteritis without a temporal artery biopsy
}

\author{
Keith Soo Keat Ong ${ }^{1}$, Lawrence Tsng Chze Ong ${ }^{2}$, Lillian Beatrice Ong ${ }^{3}$ \\ 'Ophthalmologist, RNSH-Ryde Hospital, Dalcross Adventist Hospital, University \\ of Sydney; ${ }^{2}$ Immunology Registrar, Westmead Hospital; ${ }^{3} \mathrm{JMO}$, Prince of Wales \\ Hospital, Australia
}

We would like to report 2 cases of suspected temporal arteritis which were managed without routine temporal artery biopsy.

Temporal Arteritis, otherwise known as Giant Cell Arteritis or Cranial Arteritis, is a large vessel vasculitis that primarily affects the cranial arteries and commonly presents with symptoms such as headache, fever, malaise, jaw claudication, polymyalgia rheumatica, or visual symptoms (diplopia, blurred vision, amaurosis fugax or visual loss). Because delays in treatment can result in blindness, timely corticosteroid treatment is paramount. However, the risk of significant corticosteroid side effects necessitates an accurate diagnosis. The gold standard diagnostic test for temporal arteritis, the temporal artery biopsy (TAB), is known to be highly specific for a diagnosis of temporal arteritis, but is only positive in up to $82 \%$ of temporal arteritis cases (with sensitivities reported as low as $20 \%$. $^{1}$ This figure has been shown to decrease even further (12\%) following corticosteroid therapy.

Although it is a relatively minor procedure, the risks of TAB are not trivial and include facial nerve injury, infection, haemorrhage, incisional alopecia, scar widening and foreign body reaction to entrapped hairs. ${ }^{2}$ Furthermore, the need for theatre time and skilled personnel in a procedure with a significant number of false negatives makes TAB questionable.

\section{Case 1}

An 83 year-old male presented with diplopia due to right esotropia of about 25 prism dioptres. He had no headache, jaw claudication, or other visual symptoms such as reduced visual acuity or visual field defect. Ocular movements were consistent with a right cranial nerve VI paresis. Visual acuity was 6/9 in each eye.

$\mathrm{He}$ had a history of glaucoma and was not on any medication after previous Selective Laser Trabeculoplasty. He also had a history of atrial fibrillation, hyperlipidaemia, hypertension, sleep apnoea, coronary artery stent and bilateral cataract surgery. Carotid artery duplex did not show any haemodynamically significant stenosis.

His erythrocyte sedimentation rate (ESR) on presentation was 90 and C-reactive protein (CRP) was 50.5. His clinical presentation suggested a moderate likelihood of Temporal Arteritis. Temporal artery biopsy was discussed and the patient elected to not have it as he had an upper respiratory tract infection and wished to avoid

Correspondence: Keith Ong, Sydney, Australia.

E-mail: keithong@optusnet.com.au 
any invasive procedures. A trial of Prednisone was commenced at $75 \mathrm{mg}$ daily. ESR after 17 days was 4 and CRP was 0.4 . When he was seen on day 25, his diplopia had resolved, hence the prednisone dose was reduced. However, on day 35, ESR had increased to 93 and CRP was 14.6. Hence, prednisone was recommenced at $75 \mathrm{mg}$ daily. He had no further symptoms.

In this case, the diplopia consistent with right cranial nerve VI paresis may be due to ischaemic necrosis of the extraocular muscles or ischaemia of the nerve itself ${ }^{3}$.

Although the symptoms and biochemical changes were highly suggestive of Temporal Arteritis, a rheumatological review was sought to exclude other possible causes of this clinical picture. The patient was subsequently commenced on a tapering corticosteroid regimen. To date, no other causes have been found for this patient's elevated ESR and CRP.

\section{Case 2}

An 82 year-old man presented with blurred vision of his right eye and eye ache/headache. His past medical history included diabetes, hypertension and hyperlipidaemia.

On examination, vision of the right eye was 6/18; in the left eye 6/9. Humphrey visual field test showed a small inferior paracentral visual field defect (Figure 1). ESR at presentation was 5, CRP 0.4. Fundus examination showed swelling and haemorrhage of the superior optic disc, likely consistent with AION (Figures 2 and 3). Temporal artery biopsy was suggested, but the patient declined as it was "not going to make him better". He was commenced on prednisone $50 \mathrm{mg}$ daily.
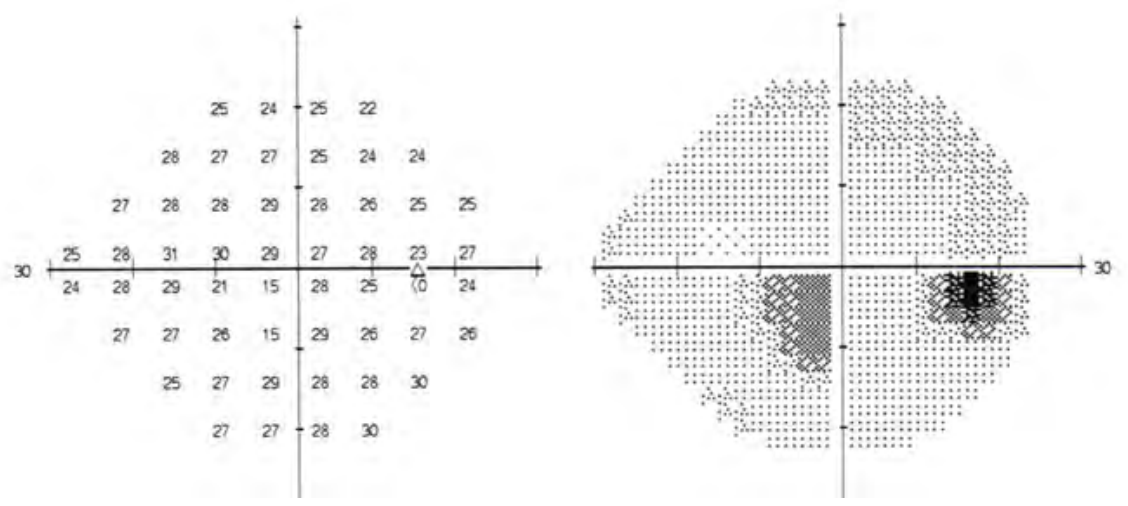

Figure 1: Right eye Humphrey Visual Field Test at presentation

On Day 22 his right eye vision had improved to 6/12, he no longer complained of visual loss in the right eye, and visual field testing was normal. The optic disc haemorrhage appeared to be resolving. On Day 43 the haemorrhage had resolved. He had no headaches or visual symptoms. Vision was $6 / 12$ in both eyes due to cataracts. Prednisone was reduced and eventually ceased. After 4 months, visual 
acuity was 6/9 part in each eye, there was no disc haemorrhage, cup/disc ratios were 0.4 in the right eye and 0.5 in the left eye. Humphrey visual field test was normal.

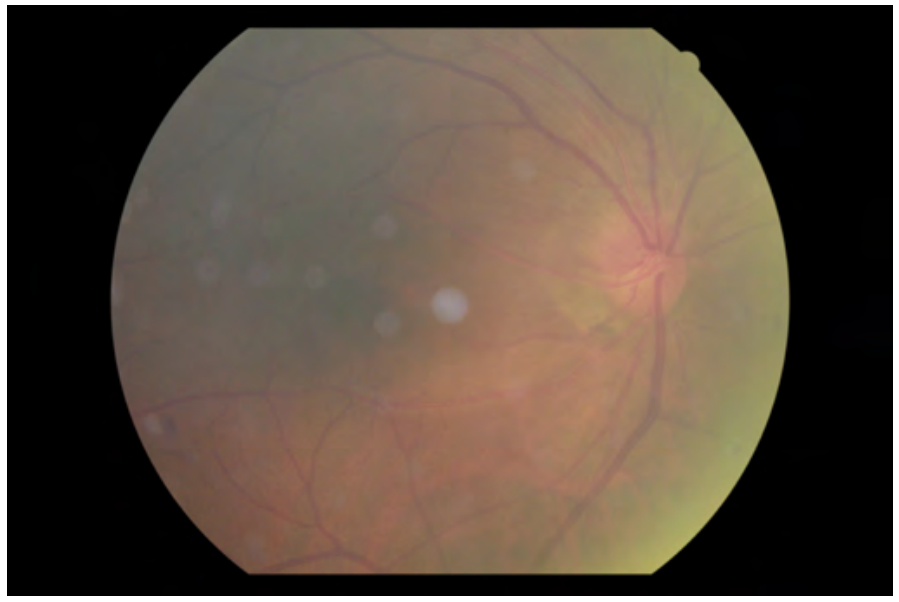

Figure 2: Day 1 swelling of superior optic disc.

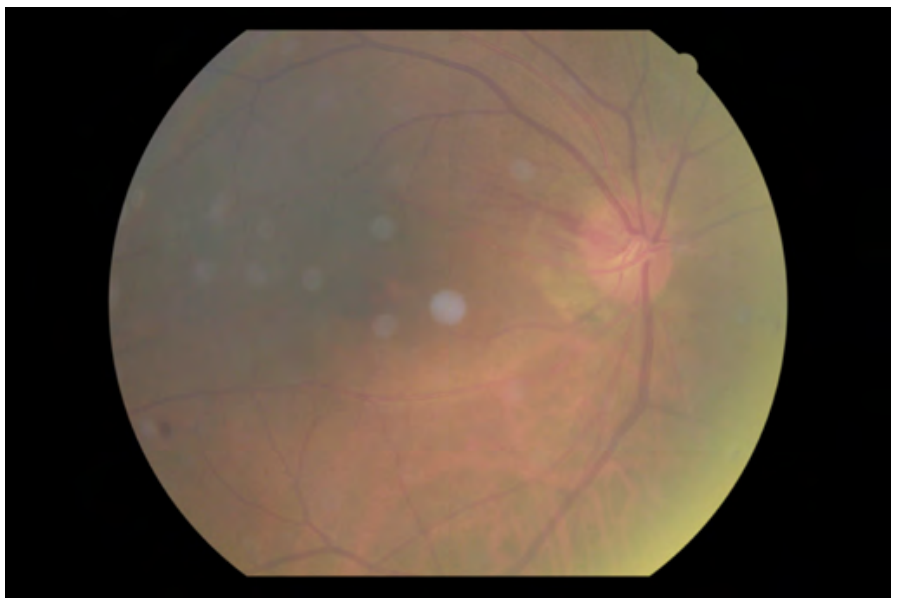

Figure 3: Day 3 optic disc margin haemorrhage.

This patient had right AION. This may be arteritic or non-arteritic. The dramatic improvement with prednisone suggests an arteritic process, although ESR and CRP were normal. Carotid dopplers showed no haemodynamically significant stenosis in the carotid arteries.

Corticosteroids would have been commenced regardless of the aetiology of AION. $^{4}$

\section{Discussion}

Even if TAB were performed, a high index of suspicion would have led to commencement of corticosteroid therapy regardless of the biopsy result. The only drawback 
in not conducting a TAB appears to be the lack of additional conclusive evidence supporting a diagnosis of temporal arteritis.

The two cases presented here illustrate how a trial of corticosteroid therapy is a reasonable and practical option for patients who prefer not to have a TAB. The strength and duration of corticosteroid therapy will still be guided by clinical progress as well as biochemical markers such as ESR and CRP. As the result of TAB does not significantly alter the management of suspected TA, perhaps it is time to review and form a consensus on the indications for temporal artery biopsy for suspected temporal arteritis.

\section{References}

1. Davies C, Frost B, Eshan O, McLain A, Shandall A. Temporal artery biopsy... Who needs one? Postgrad Med J. 2006;82(969):476-8.

2. Winkler A, Wudel J. Medscape - Temporal Artery Biopsy 2012 [9th September 2012]; Available from: http://emedicine.medscape.com/article/1520091-overview\#a17.

3. Barricks M, Traviesa D, Glaser J, Levy I. Ophthalmoplegia in cranial arteritis. Brain. 1977;100(2):209-21.

4. Hayreh SS. Vascular disorders in neuro-ophthalmology. Current Opinion in Neurology February. 2011;24(1):6-11. 